\title{
DIFFRACTIVE INTERACTION OF DEUTERONS AND THREE-NUCLEON-PARTICLES WITH HEAVY NUCLEI
}

\author{
Yu. A. Berezhnoy ${ }^{1}$, V. Yu. Korda ${ }^{2}$ \\ ${ }^{1}$ Kharkiv State University, Kharkiv, UA-310077, Ukraine \\ ${ }^{2}$ Scientific and Technological Center of Electrophysics, \\ National Academy of Sciences of Ukraine, \\ 28 Chernyshevs'kyi Str., P. O. BOX 8812, Kharkiv, UA-310002, Ukraine \\ (Received October 13, 1997; received in final form March 16, 1998)
}

\begin{abstract}
The approach which provides for the analytical expressions for the elastic and inelastic scattering and dissociation-into-two and -three-parts differential cross-sections of deuterons and threenucleon-particles is developed. The dissociation cross-sections are the oscillating functions of the scattering angle of the projectile center-of-mass. The analysis of experimental data on the elastic and inelastic scattering of deuterons and ${ }^{3} \mathrm{He}-$ nuclei by heavy nuclei at intermediate energies is performed. The results of calculations are in good agreement with the measured differential crosssections for all the target nuclei considered.
\end{abstract}

Key words: diffraction model, differential cross-section, elastic and inelastic scattering and dissociation.

PACS numbers: 24.10.Ht, 25.45.-z, 25.45.De

\section{INTRODUCTION}

Deuterons, ${ }^{3} \mathrm{H}$ and ${ }^{3} \mathrm{He}$ are the simplest composite weakly bound nuclear particles. Therefore, these light nuclei are easily broken up into two and three parts in the field of heavy nuclei, which leads to a greater variety of final channels than in point particle scattering.

The experimental data on elastic scattering of light nuclei by the nuclei at intermediate energies are usually analyzed with the help of complex optical potential [1][3]. The simplest Saxon-Woods optical potential contains six parameters (three for the real part and three for the imaginary one). The data obtained this way provides important information about the nuclear structure and the absorptive and refractive properties of nuclear matter with respect to the projectiles. However, this approach has a deficiency in that the inner structure of composite nuclei is not taken into account in the conventional optical model.

The alternative method of analyzing the experimental data of the intermediate energy particles elastically scattered by nuclei is based on the use of the theory of diffraction interaction of light nuclei with heavy nuclei. In such an approach the projectile inner structure is considered, while the heavy target nucleus is treated as the structureless matter with definite absorptive and refractive properties, similarly to the optical model.

The differential cross-sections of diffraction light nuclei elastic scattering are determined by the radii of colliding particles (the effective interaction radius), the target nucleus surface diffuseness value and the nuclear surface refraction coefficient. This means that such crosssections are determined by the geometric characteristics of the collision conditioned by the quasiclassic character of the considered processes.

Diffraction theory makes it possible to derive a simple expression for the differential cross-section of the elastic scattering of complex particles by the nuclei in the approximation where the linear size of the projectile is small as compared with the one for the target nucleus. In this approach the inner structure of light composite particle is taken into consideration and the cross-section contains only three fitting parameters, twice as less as for the cross section in the optical model. Although the considered approach is valid only for the heaviest nuclei, in fact it appears suitable also for analyzing the scattering of composite particles by medium weight target nuclei.

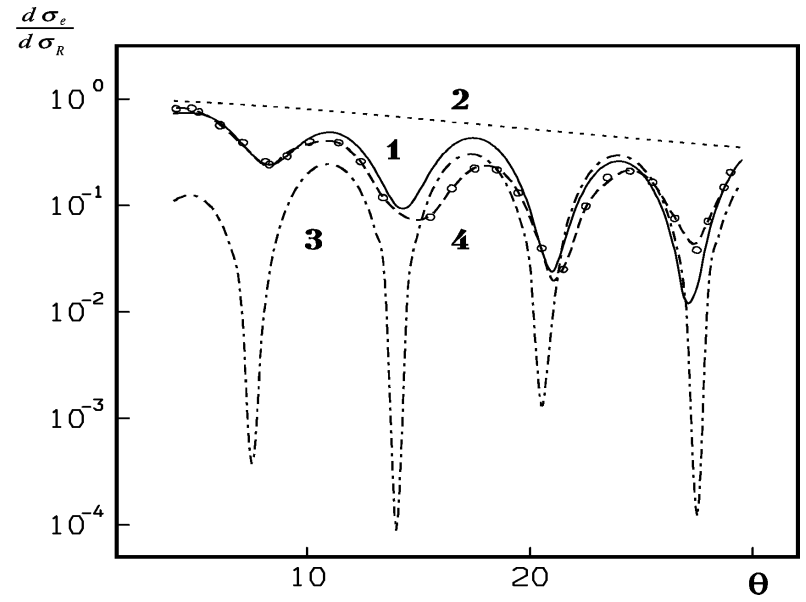

Fig. 1. The ratio of the elastic scattering differential cross-section for $110 \mathrm{MeV}$ deuterons on ${ }^{208} \mathrm{~Pb}(R=7.9 \mathrm{fm}$, $d=0.55 \mathrm{fm}, \gamma=0.12$ ) to the Rutherford one, as a function of the scattering angle $\theta$ (deg.). Curve 1 shows the interference between the Coulomb elastic scattering and the nuclear one. Curve 2 is for pure Coulomb elastic scattering. Curve 3 is for pure nuclear elastic scattering. Curve 4 is the optical model calculations taken from [3]. 
The diffraction approach was applied to the deuteronnucleus interaction in [4]- [6]. In [7] the general diffraction theory was built. This theory was extended on the case of interaction between three-nucleon-particles and nuclei [8]. The three-nucleon-particle-nuclei interaction was analyzed in [9] on the basis of the diffraction model in the $R_{t} \ll R$ approximation ( $R_{t}$ is the radius of three-nucleon-particle and $R$ is the nuclear radius) where the profile functions were expanded into a series. The role of the nuclear surface diffuseness consideration in deuteron-nucleus elastic and inelastic scattering was clarified in [10].

Therefore, it appears valuable to develop the general diffraction approach which could enable to obtain closedform expressions for the elastic and inelastic scattering and dissociation-into-two and -three-parts cross sections for the two- and three-nucleon-particle-nuclei interaction at intermediate energies with the allowance for the Coulomb interaction, the inner structure of the projectile, the nuclear surface diffuseness and the nuclear matter refraction.

\section{AMPLITUDE OF THE DEUTERON-NUCLEUS INTERACTION}

In this section we calculate the amplitude of the deuteron-nucleus scattering with the excitation of low lying vibrational states of nuclei with the allowance for the Coulomb interaction, the inner deuteron structure, the finite values of nuclear surface diffuseness and surface refraction, with neglect of spins and isospins of the nucleons.

The amplitude of the deuteron-nucleus interaction is defined by formula [7]

$$
F(\mathbf{q})=\int d^{3} r \varphi_{f}^{*}(\mathbf{r}) F(\mathbf{q}, \mathbf{s}) \varphi_{0}(\mathbf{r})
$$

where $\mathbf{r}=\mathbf{r}_{p}-\mathbf{r}_{n}$ is the distance between the nucleons in the deuteron; $\mathbf{r}_{n}$ and $\mathbf{r}_{\mathbf{p}}$ are the neutron and proton radius-vectors; $\mathbf{s}$ is the projection of the vector $\mathbf{r}$ on the plane perpendicular to the incident deuteron beam direction ( $z$ - axis); $\mathbf{q}$ is the momentum transfer of the deuteron center-of-mass; $\varphi_{0}(\mathbf{r})$ is the deuteron groundstate wavefunction; $\varphi_{f}(\mathbf{r})$ is the wavefunction of the related motion of the nucleons, the deuteron was consisted, in the final state. The amplitude $F(\mathbf{q}, \mathbf{s})$ is equal to

$$
\begin{aligned}
F(\mathbf{q}, \mathbf{s}) & =\frac{i K}{2 \pi} \int d^{2} b \exp (i \mathbf{q b}) \\
& \times[1-\exp [2 i \chi(\mathbf{b}, \mathbf{s})]]
\end{aligned}
$$

where $K$ is the projectile wavevector; $\mathbf{b}=\left(\mathbf{b}_{n}+\mathbf{b}_{p}\right) / 2$ is the impact parameter of the deuteron center-of-mass (we consider the neutron and proton masses to be equal); $\mathbf{b}_{n}$ and $\mathbf{b}_{p}$ are the neutron and proton impact parameters; $\chi(\mathbf{b}, \mathbf{s})$ is the scattering phase of deuteron. The deuteron scattering phase has the form

$$
\chi(\mathbf{b}, \mathbf{s})=\chi_{n}\left(\mathbf{b}_{n}\right)+\chi_{p}\left(\mathbf{b}_{p}\right)+\sigma_{p}\left(\mathbf{b}_{p}\right),
$$

where $\mathbf{b}_{n, p}=\mathbf{b} \pm \mathbf{s} / 2, \chi_{n, p}\left(\mathbf{b}_{n, p}\right)$ are the nuclear parts of the neutron and proton scattering phases; $\sigma_{p}\left(\mathbf{b}_{p}\right)$ is the Coulomb part of the proton scattering phase.

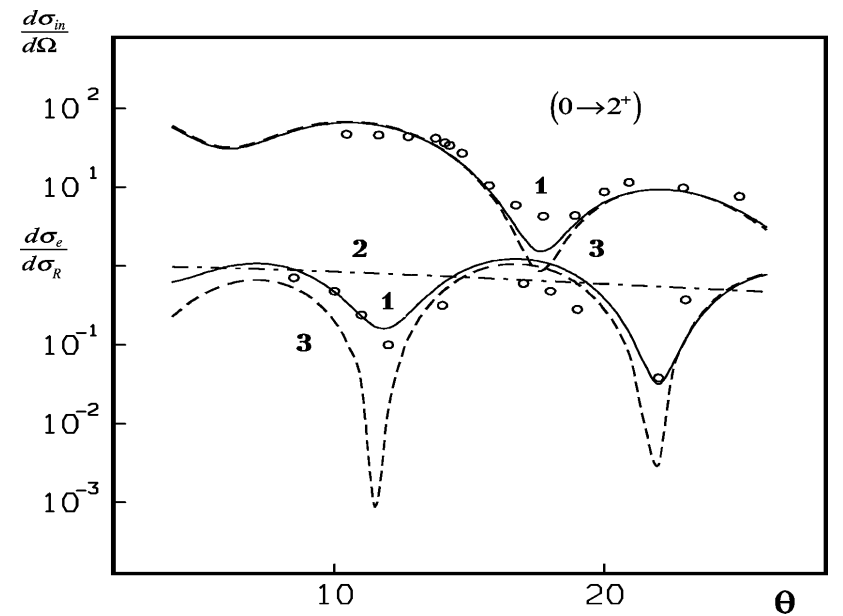

Fig. 2. The inelastic scattering differential cross-section $(\mathrm{mb} / \mathrm{sr})$ with the excitation of the first $2^{+}$state (the upper figure) and the ratio of the elastic scattering differential cross-section to the Rutherford one (the lower figure) for the $80 \mathrm{MeV}$ deuterons on the ${ }^{68} \mathrm{Zn}$-nuclei $(R=5.7 \mathrm{fm}$, $\left.d=0.60 \mathrm{fm}, \gamma=0.18, \beta_{2}=0.25\right)$. Curves 1 show the interference between the Coulomb scattering and the nuclear one. Curve 2 is for pure Coulomb scattering. Curves 3 are for pure nuclear scattering. Experimental data are taken from [20].

The amplitude $F(\mathbf{q}, \mathbf{s})$ can be presented [11] as a sum of the Coulomb $F_{C}(\mathbf{q}, \mathbf{s})$ and the nuclear scattering amplitudes distorted by the Coulomb interaction $F_{N}(\mathbf{q}, \mathbf{s})$ :

$$
\begin{gathered}
F(\mathbf{q}, \mathbf{s})=F_{C}(\mathbf{q}, \mathbf{s})+F_{N}(\mathbf{q}, \mathbf{s}), \\
F_{C}(\mathbf{q}, \mathbf{s})=2 f_{C}\left(\frac{K}{2}, q\right) \exp (i \mathbf{q s} / 2),
\end{gathered}
$$

$$
\begin{aligned}
F_{N}(\mathbf{q}, \mathbf{s}) & =\frac{i K}{2 \pi} \int d^{2} b \exp \left(i \mathbf{q b}+2 i \sigma_{p}\left(\mathbf{b}_{p}\right)\right) \\
& \times\left[1-\exp \left(2 i\left(\chi_{n}\left(\mathbf{b}_{n}\right)+\chi_{p}\left(\mathbf{b}_{p}\right)\right)\right)\right],
\end{aligned}
$$

$$
f_{C}\left(\frac{K}{2}, q\right)=-K R^{2} \frac{n \Gamma(1+i n)}{\Gamma(1-i n)} \frac{\exp (-2 i n \ln (q / K))}{(q R)^{2}}
$$




$$
\exp \left(2 i \sigma_{p}\left(\mathbf{b}_{p}\right)\right)=\frac{\Gamma(1+K b / 2+i n)}{\Gamma(1+K b / 2-i n)}
$$

where $n=\frac{2 M Z e^{2}}{\hbar^{2} K}$ is the Coulomb parameter of the deuteron's proton; $M$ is the nucleon mass; $Z e^{2}$ is the nucleus charge.

The neutron and proton profile functions $\omega_{n, p}\left(\mathbf{b}_{n, p}\right)$ are introduced by the correlations

$$
\omega_{n, p}\left(\mathbf{b}_{n, p}\right)=1-\exp \left(2 i \chi_{n, p}\left(\mathbf{b}_{n, p}\right)\right)
$$

Therefore, the amplitude $F_{N}(\mathbf{q}, \mathbf{s})$ has the form

$$
\begin{aligned}
F_{N}(\mathbf{q}, \mathbf{s}) & =\frac{i K}{2 \pi} \int d^{2} b \exp \left(i \mathbf{q} \mathbf{b}+2 i \sigma_{p}\left(\mathbf{b}_{p}\right)\right) \\
& \times \omega_{d}(\mathbf{b}, \mathbf{s})
\end{aligned}
$$

$$
\omega_{d}(\mathbf{b}, \mathbf{s})=\omega_{n}\left(\mathbf{b}_{n}\right)+\omega_{p}\left(\mathbf{b}_{p}\right)-\omega_{n}\left(\mathbf{b}_{n}\right) \omega_{p}\left(\mathbf{b}_{p}\right)
$$

The magnitudes $\omega_{n, p}\left(\mathbf{b}_{n, p}\right)$ can be chosen as [12]

$$
\omega_{n}\left(b_{n}\right)=\omega_{p}\left(b_{p}\right)=\omega(b)=\left[1+\exp \left(\frac{b-R}{d}+i \gamma\right)\right]^{-1},
$$

where $d$ is the nuclear surface diffuseness value $(d \ll R)$; $\gamma$ characterizes the refraction of the scattered wave on the nuclear edge. The nucleon-nucleus profile functions in the form (12) enables one to explain the nucleon-nucleus interaction at intermediate energies [13].

Further calculations will be accomplished in the $R_{d} \ll$ $R$ approximation, where $R_{d}$ is the deuteron radius. We shall assume that the shape of the nucleus in the excited state is described by the expression

$$
R(\vartheta, \psi)=R\left\{1+\sum_{l, m} \alpha_{l, m} Y_{l, m}(\vartheta, \psi)\right\}
$$

where $R$ is the radius of the spherical nucleus of the same volume, $\alpha_{l, m}$ are the nuclear deformation parameters and $Y_{l, m}(\vartheta, \psi)$ are the spherical functions.

The projection of the nucleus on the plane perpendicular to the direction of the incident deuteron beam within the accuracy up to the linear terms over the nuclear deformation parameters $\alpha_{l, m}$ is given by the formula

$$
R\left(\frac{\pi}{2}, \psi\right)=R\left\{1+\sum_{l, m} \alpha_{l, m} Y_{l, m}\left(\frac{\pi}{2}, \psi\right)\right\}
$$

Considering the deformation parameters as small values we expand the profile function $\omega(b)$ within the accu- racy up to linear terms in $\alpha_{l, m}$ :

$$
\omega(b)=\left\{1+\sum_{l, m} \alpha_{l, m} Y_{l, m}\left(\frac{\pi}{2}, \psi\right) R \frac{\partial}{\partial R}\right\} \omega_{0}(b),
$$

where $\omega_{0}(b)$ is defined by formula (12).

Substituting (15) into formula (11) and then expanding $\omega_{d}(\mathbf{b}, \mathbf{s})$ within the accuracy up to the linear terms in $\alpha_{l, m}$, we find

$$
\omega_{d}(\mathbf{b}, \mathbf{s})=\left\{1+\sum_{l, m} \alpha_{l, m} Y_{l, m}\left(\frac{\pi}{2}, \psi\right) R \frac{\partial}{\partial R}\right\} \omega^{(d)}(\mathbf{b}, \mathbf{s}),
$$

where $\omega^{(d)}(\mathbf{b}, \mathbf{s})$ is defined by the formula (11).

The deuteron-nucleus profile function $\omega^{(d)}(\mathbf{b}, \mathbf{s})$ can be represented in the form

$$
\omega^{(d)}(\mathbf{b}, \mathbf{s})=\left(1+d \frac{\partial}{\partial R}\right) \omega_{1}(\mathbf{b}, \mathbf{s})+\omega_{2}(\mathbf{b}, \mathbf{s})
$$

$$
\begin{aligned}
& \omega_{1}(\mathbf{b}, \mathbf{s})=\frac{1}{2}\left[\omega_{n}\left(\mathbf{b}_{n}\right)+\omega_{p}\left(\mathbf{b}_{p}\right)\right] \\
& \omega_{2}(\mathbf{b}, \mathbf{s})=\frac{1}{2}\left[\omega_{n}\left(\mathbf{b}_{n}\right)-\omega_{p}\left(\mathbf{b}_{p}\right)\right]^{2},
\end{aligned}
$$

where it is noted that for the profile function (12) the following relation is valid:

$$
\omega^{2}(b)=\left(1-d \frac{\partial}{\partial R}\right) \omega(b)
$$

Therefore, amplitude (10) is equal to

$$
F_{N}(\mathbf{q}, \mathbf{s})=F_{0}(\mathbf{q}, \mathbf{s})+\sum_{l, m} \alpha_{l, m} Y_{l, m}\left(\frac{\pi}{2}, 0\right) R \frac{\partial}{\partial R} F_{m}(\mathbf{q}, \mathbf{s})
$$

$$
F_{j}(\mathbf{q}, \mathbf{s})=\left(1+d \frac{\partial}{\partial R}\right) F_{j}^{(1)}(\mathbf{q}, \mathbf{s})+F_{j}^{(2)}(\mathbf{q}, \mathbf{s})
$$

$$
F_{j}^{(1)}(\mathbf{q}, \mathbf{s})=\frac{1}{2}\left[\exp \left(i \frac{\mathbf{q} \mathbf{s}}{2}\right)+\exp \left(-i \frac{\mathbf{q} \mathbf{s}}{2}\right)\right] f_{j}(\mathbf{q})
$$




$$
\begin{aligned}
F_{j}^{(2)}(\mathbf{q}, \mathbf{s}) & =\frac{i K}{2 \pi} \int d^{2} b \exp \left(i \mathbf{q} \mathbf{b}+2 i \sigma_{p}\left(\mathbf{b}_{p}\right)\right. \\
& \left.+i m \psi \delta_{j, m}\right) \omega_{2}(\mathbf{b}, \mathbf{s}),
\end{aligned}
$$

where $j=0, m ; \delta_{i, j}=0$ if $i \neq j$ and $\delta_{i, j}=1$ if $i=j$.

The amplitude of nucleon-nucleus interaction has the form

$$
\begin{aligned}
f_{j}(\mathbf{q}) & =\frac{i K}{2 \pi} \int d^{2} b \exp \left(i \mathbf{q} \mathbf{b}+2 i \sigma_{p}(\mathbf{b})\right. \\
& \left.+i m \psi \delta_{j, m}\right) \omega_{0}(\mathbf{b})
\end{aligned}
$$

where $\mathbf{b}_{p}$ in the Coulomb phase is replaced by $\mathbf{b}$ in the $R_{d} \ll R$ approximation.

As the amplitudes $F_{j}^{(2)}(\mathbf{q}, \mathbf{s})$ turn into zero for the point deuteron $F_{j}^{(2)}(\mathbf{q}, 0)=0$, i.e. they are proportional to $R_{d}$, they should be calculated by the formula

$$
F_{j}^{(2)}(\mathbf{q}, \mathbf{s})=\Phi(q)\left[F_{j}^{(2)}(\mathbf{q}, \mathbf{s})\right]_{d=0},
$$

where the damping factor $\Phi(q)$ is equal to [14]

$$
\Phi(q)=\frac{\pi q d}{\sinh (\pi q d)}
$$

The amplitudes $F_{j}^{(2)}(\mathbf{q}, \mathbf{s})$ can be estimated in the following way. When $\mathrm{d}=0$, the neutron and proton profile functions are equal to: $\omega_{n, p}(b<R)=1, \omega_{n, p}(b>R)=0$.

So far as the deuteron ground-state wavefunction decreases rapidly when the distance between the neutron and the proton increases, the absolute values of the impact parameters $b_{n, p}$ can be expanded into a series within the accuracy up to the linear in $s$ terms: $b_{n, p}=$ $b \pm(s / 2) \cos \varphi$, where $\varphi$ is the angle between the vectors $\mathbf{b}$ and $\mathbf{s}$. Since $b \sim R$ and $|\mathbf{s}| \sim R_{d}$, this decomposition is equivalent to the $R_{d} \ll R$ approximation. Therefore, the profile functions $\omega_{n, p}\left(\mathbf{b}_{n, p}\right)$ can be presented in the form: $\omega_{n, p}\left(b_{n, p}\right)=1$, when $b<R \mp(s / 2) \cos \varphi, \omega_{n, p}\left(b_{n, p}\right)=0$ when $b>R \mp(s / 2) \cos \varphi$.

As the amplitudes $F_{j}^{(2)}(\mathbf{q}, \mathbf{s})$ contain the combinations $\left[\omega_{n}\left(b_{n}\right)-\omega_{p}\left(b_{p}\right)\right]^{2}$ we have to find the modulus of difference between the neutron and proton profile functions:

$$
\left|\omega_{n}\left(b_{n}\right)-\omega_{p}\left(b_{p}\right)\right|=\left\{\begin{array}{l}
0, b<R_{-}(s, \varphi) \\
1, R_{-}(s, \varphi) \leq b \leq R_{+}(s, \varphi) \\
0, b>R_{+}(s, \varphi)
\end{array}\right\}
$$

where the notations introduced are $R_{ \pm}(s, \varphi)=R \pm$ $(s / 2)|\cos \varphi|$. Thus, the magnitude $\left|\omega_{n}\left(b_{n}\right)-\omega_{p}\left(b_{p}\right)\right|$ is a step the width of which is determined by the deuteron radius $R_{d}$.

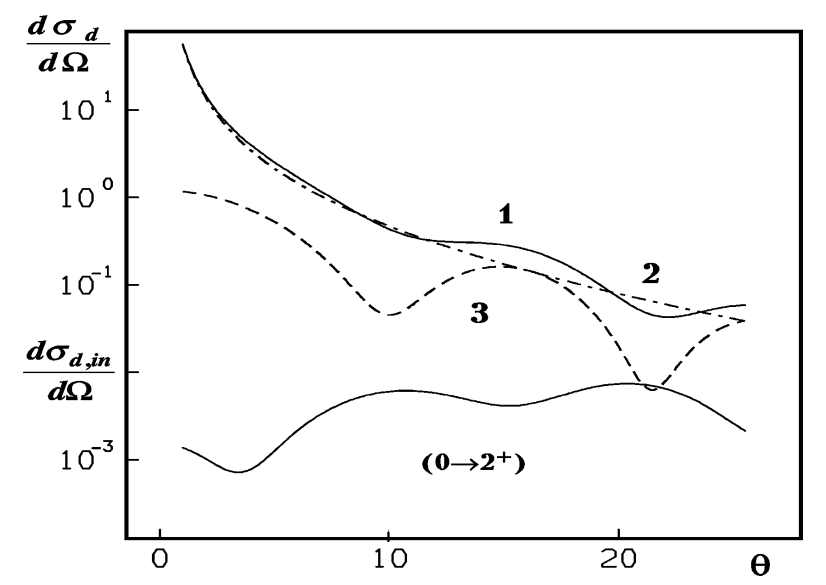

Fig. 3. The differential cross-section $(b / s r)$ of the $80 \mathrm{MeV}$ deuterons dissociated without (the upper figure) and with the excitation of the first $2^{+}$state (the lower figure) of the ${ }^{68} \mathrm{Zn}$-nuclei $\left(R=5.7 \mathrm{fm}, d=0.60 \mathrm{fm}, \gamma=0.18, \beta_{2}=0.25\right)$. Curve 1 shows the interference between the Coulomb dissociation and the nuclear one. Curve 2 is for pure Coulomb dissociation. Curve 3 is for pure nuclear dissociation.

Integrating in formulae (22) with the allowance for (24), (26) and expanding the derived expressions up to the linear in $s$ terms, we find

$$
F_{j}^{(2)}(\mathbf{q}, \mathbf{s})=i K R^{2} \Phi(q) \frac{s|\cos \varphi|}{2 R} J_{|j|}(q R) \exp \left(2 i \sigma_{p}(R)\right)
$$

Within the accuracy of up to the linear terms in $s / R$, amplitudes (20) are equal to

$$
\begin{aligned}
F_{j}(\mathbf{q}, \mathbf{s}) & =\frac{1}{2}\left[\exp \left(i \frac{\mathbf{q} \mathbf{s}}{2}\right)+\exp \left(-i \frac{\mathbf{q s}}{2}\right)\right]\left(1+d \frac{\partial}{\partial R}\right) f_{j}(\mathbf{q}) \\
& +i K R^{2} \Phi(q) \frac{s|\cos \varphi|}{2 R} J_{|j|}(q R) \exp \left(2 i \sigma_{p}(R)\right) .
\end{aligned}
$$

So far as the inelastic interaction of deuterons with the excitation of the low lying vibrational levels of the nucleus 
has the surface character and the Coulomb phase slightly changes on the nucleus edge, the Coulomb phase in the expression for $F_{m}(\mathbf{q}, \mathbf{s})$ can be treated as constant [15], [16].

Now amplitude (1) acquires the form

$$
\begin{gathered}
F(\mathbf{q})=F_{e}(\mathbf{q})+\sum_{l, m} \alpha_{l, m} Y_{l, m}\left(\frac{\pi}{2}, 0\right) F_{m}(\mathbf{q}) \\
F_{e}(\mathbf{q})=2 f_{C}\left(\frac{K}{2}, q\right) S\left(\frac{\mathbf{q}}{2}\right)+\frac{1}{2}\left[S\left(\frac{\mathbf{q}}{2}\right)+S\left(-\frac{\mathbf{q}}{2}\right)\right]\left(1+d \frac{\partial}{\partial R}\right) f_{0}(\mathbf{q}) \\
+i K R^{2} p \Phi(q) J_{0}(q R) \exp \left(2 i \sigma_{p}(R)\right), \\
F_{m}(\mathbf{q})=\frac{1}{2}\left[S\left(\frac{\mathbf{q}}{2}\right)+S\left(-\frac{\mathbf{q}}{2}\right)\right] R \frac{\partial}{\partial R}\left(1+d \frac{\partial}{\partial R}\right) f_{m}(\mathbf{q}) \\
+i K R^{2} p \Phi(q)\left(q R J_{|m|-1}(q R)-(|m|-1) J_{|m|}(q R)\right) \exp \left(2 i \sigma_{p}(R)\right) . \\
S(\mathbf{q})=\int d^{3} r \exp (i \mathbf{q s}) \varphi_{f}^{*}(\mathbf{r}) \varphi_{0}(\mathbf{r}), Q=2 \int d^{3} r s|\cos \varphi| \varphi_{f}^{*}(\mathbf{r}) \varphi_{0}(\mathbf{r}), p=Q /(4 R) .
\end{gathered}
$$

\section{DIFFERENTIAL CROSS-SECTIONS OF THE DEUTERON-NUCLEUS INTERACTION}

We choose the deuteron ground-state wavefunction in the form

$$
\varphi_{0}(r)=\sqrt{\frac{\alpha}{2 \pi}} \frac{\exp (-\alpha r)}{r}
$$

where $\alpha=\sqrt{m \epsilon} / \hbar, \epsilon$ is the deuteron binding energy, $\alpha=0.232 \mathrm{fm}^{-1}\left(R_{d}=1 / 2 \alpha\right)$.

Wavefunction (32) is normalized to unity and corresponds to the zero-value nuclear force range assumption (the $D$ - wave admixture to the deuteron ground-state is neglected).

If the deuteron dissociation takes place, the related motion of the neutron and proton released from the deuteron dissociation is described by the wavefunction (the zero-value nuclear force assumption is assumed)

$$
\varphi_{\mathbf{f}}(\mathbf{r})=\exp (i \mathbf{f r})+\frac{1}{i f-\alpha} \frac{\exp (-i f r)}{r}
$$

where $\mathbf{f}=\left(\mathbf{k}_{p}-\mathbf{k}_{n}\right) / 2$ is the neutron-proton related motion wavevector, $\mathbf{k}_{n}$ and $\mathbf{k}_{p}$ are the neutron and proton wavevectors in the final state. The wavefunctions (32) and (33) are orthogonal and make a complete set of functions.

Applying the wavefunction (32) as $\varphi_{f}(\mathbf{r})$ and calculating the integral in formulae (31), we find

$$
S_{e}(\mathbf{q})=\frac{1}{q R_{d}} \arctan \left(q R_{d}\right), Q_{e}=R_{d}
$$

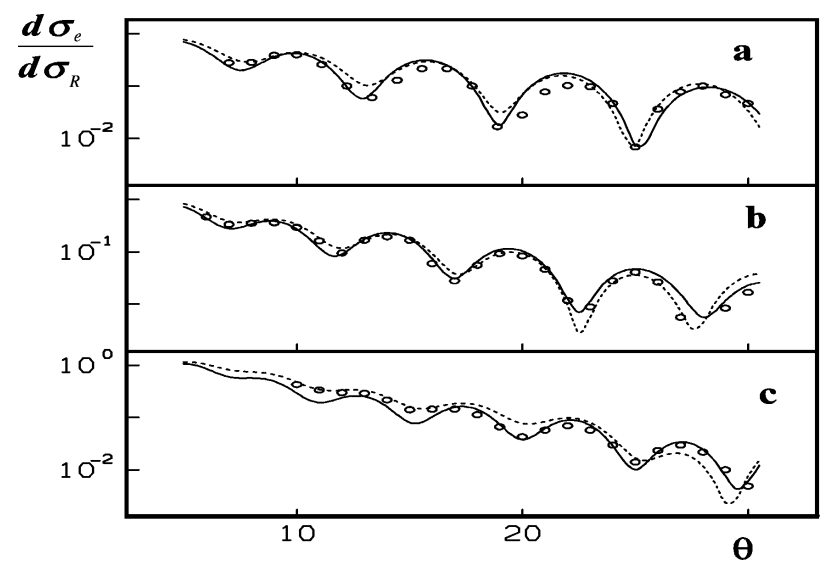

Fig. 4. The ratio of the elastic scattering differential cross-section for the $130 \mathrm{MeV}{ }^{3} \mathrm{He}-$ nuclei on ${ }^{90} \mathrm{Zr}$ (a), ${ }^{120} \mathrm{Sn}$ (b) and ${ }^{208} \mathrm{~Pb}$ (c) nuclei, to the Rutherford one, as a function of the scattering angle $\theta$ (deg.). Solid lines are for the composed ${ }^{3} \mathrm{He}-$ nuclei, while dashed lines are for the quasipoint ${ }^{3} \mathrm{He}-$ nuclei. Experimental data are taken from [1].

Calculating the integral in formula (31) with wave- 
function (33) taken as $\varphi_{f}(\mathbf{r})$, we obtain

$$
\begin{gathered}
S_{d}(\mathbf{q}, \mathbf{f})=\sqrt{8 \pi \alpha}\left\{\frac{1}{\alpha^{2}+(\mathbf{q}-\mathbf{f})^{2}}\right. \\
\left.+\frac{f+i \alpha}{2 q\left(\alpha^{2}+f^{2}\right)} \ln \frac{f-q+i \alpha}{f+q+i \alpha}\right\}, \\
Q_{d}(\mathbf{f})=\frac{4 \sqrt{2 \pi \alpha}}{P\left(\alpha^{2}+f^{2}\right)^{2}}\left[P^{2}-f_{x}^{2}-\frac{1}{2} P(\alpha+i f)\right], \\
P^{2}=\alpha^{2}+f^{2}-f_{x}^{2}
\end{gathered}
$$

where $f_{x}=f_{\perp} \cos \beta, \mathbf{f}_{\perp}$ is the projection of the vector f on the plane perpendicular to the incident deuteron beam direction, and $\beta$ is the angle between the vectors $\mathbf{q}$ and $\mathbf{f}_{\perp}$.

The differential cross-sections of elastic scattering and inelastic scattering with the excitation of one-phonon vibrational states with spin $I$ in even-even nuclei are given as

$$
\begin{aligned}
& \frac{d \sigma_{e}}{d \Omega}=\left|F_{e}(\mathbf{q})\right|^{2}, \\
& \frac{d \sigma(0 \rightarrow I)}{d \Omega}=\frac{\left|\beta_{I}\right|^{2}}{2 I+1} \sum_{m=-I}^{I}\left|Y_{I, m}\left(\frac{\pi}{2}, 0\right) F_{m}(\mathbf{q})\right|^{2},
\end{aligned}
$$

where the dynamical deformation parameter $\beta_{I}$ has the form $\beta_{I}=\sqrt{2 I+1}\left\langle l m\left|\alpha_{l, m}\right| 00\right\rangle$, the scattering angle $\theta$ of the neutron-proton center-of-mass is related to the momentum transfer by the correlation $q=2 K \sin (\theta / 2)$ and the amplitudes $F_{e}(\mathbf{q})$ and $F_{m}(\mathbf{q})$ are defined by formulae (30), (34). The differential cross sections of the deuteron dissociation and the deuteron dissociation with the excitation of one-phonon vibrational states with spin $I$ in even-even nuclei are equal to:

$$
\begin{aligned}
& \frac{d \sigma_{d}}{d \Omega}=\left|F_{e}(\mathbf{q}, \mathbf{f})\right|^{2} \frac{d^{3} f}{(2 \pi)^{3}} \\
& \frac{d \sigma_{d}(0 \rightarrow I)}{d \Omega}=\frac{\left|\beta_{I}\right|^{2}}{2 I+1} \sum_{m=-I}^{I}\left|Y_{I, m}\left(\frac{\pi}{2}, 0\right) F_{m}(\mathbf{q}, \mathbf{f})\right|^{2} \frac{d^{3} f}{(2 \pi)^{3}}
\end{aligned}
$$

where the amplitudes $F_{0}(\mathbf{q}, \mathbf{f})$ and $F_{m}(\mathbf{q}, \mathbf{f})$ are defined by formulae (30), (35).

The angular distributions of the neutron-proton center-of-mass, released in the deuteron dissociation and in the deuteron dissociation with the excitation of vibrational states of target-nucleus, can be obtained the following way. Cross-sections (37) with amplitude (1), integrated over the vector $\mathbf{f}$ using the completeness condition of the neutron-proton system wavefunctions $\varphi_{0}(\mathbf{r}) \varphi_{0}^{*}\left(\mathbf{r}^{\prime}\right)+\int \frac{d^{3} f}{(2 \pi)^{3}} \varphi_{\mathbf{f}}(\mathbf{r}) \varphi_{\mathbf{f}}^{*}\left(\mathbf{r}^{\prime}\right)=\delta\left(\mathbf{r}-\mathbf{r}^{\prime}\right)$, have the form

$$
\begin{aligned}
\frac{d \sigma_{d}}{d \Omega} & =\int d^{3} r|F(\mathbf{q}, \mathbf{s})|^{2}\left|\varphi_{0}(\mathbf{r})\right|^{2}-\left.\left.\left|\int d^{3} r F(\mathbf{q}, \mathbf{s})\right| \varphi_{0}(\mathbf{r})\right|^{2}\right|^{2}, \\
\frac{d \sigma_{d}(0 \rightarrow I)}{d \Omega} & =\frac{\left|\beta_{I}\right|^{2}}{2 I+1} \sum_{m=-I}^{I}\left|Y_{I, m}\left(\frac{\pi}{2}, 0\right)\right|^{2} \\
& \times\left\{\int d^{3} r\left|F_{m}(\mathbf{q}, \mathbf{s})\right|^{2}\left|\varphi_{0}(\mathbf{r})\right|^{2}-\left.\left.\left|\int d^{3} r F_{m}(\mathbf{q}, \mathbf{s})\right| \varphi_{0}(\mathbf{r})\right|^{2}\right|^{2}\right\} .
\end{aligned}
$$

Integrating in formulae (38) with the allowance for (4), (5), (19), (28), we find

$$
\begin{aligned}
\frac{d \sigma_{d}}{d \Omega} & =4\left[1-S^{2}\left(\frac{q}{2}\right)\right]\left|f_{C}\left(\frac{K}{2}, q\right)\right|^{2}+\frac{1}{2}\left\{1+S(q)-\frac{1}{2}\left[S\left(\frac{q}{2}\right)+S\left(-\frac{q}{2}\right)\right]^{2}\right\} \\
& \times\left|\left(1+d \frac{\partial}{\partial R}\right) f_{0}(q)\right|^{2}+\frac{5}{48} K^{2} R^{2} R_{d}^{2} \Phi^{2}(q) J_{0}^{2}(q R) \\
& +2\left\{1+S(q)-S\left(\frac{q}{2}\right)\left[S\left(\frac{q}{2}\right)+S\left(-\frac{q}{2}\right)\right]\right\} \operatorname{Re}\left[f_{C}\left(\frac{K}{2}, q\right)\left(1+d \frac{\partial}{\partial R}\right) f_{0}^{*}(q)\right]
\end{aligned}
$$




$$
\begin{aligned}
& -K R R_{d} \Phi(q)[1-S(q)] \operatorname{Im}\left[f_{C}^{*}\left(\frac{K}{2}, q\right) \exp \left[2 i \sigma_{p}(R)\right]\right] \\
& -\frac{1}{2} K R R_{d} \Phi(q)\left\{1-\frac{1}{2}\left[S\left(\frac{q}{2}\right)+S\left(-\frac{q}{2}\right)\right]\right\} \operatorname{Im}\left[\exp \left[2 i \sigma_{p}(R)\right]\left(1+d \frac{\partial}{\partial R}\right) f_{0}^{*}(q)\right] \\
& \frac{d \sigma_{d}(0 \rightarrow I)}{d \Omega}=\frac{\left|\beta_{I}\right|^{2}}{2 I+1} \sum_{m=-I}^{I}\left|Y_{I, m}\left(\frac{\pi}{2}, 0\right)\right|^{2} \sigma_{m}(q), \\
& \sigma_{m}(q)=\frac{1}{2}\left\{1+S(q)-\frac{1}{2}\left[S\left(\frac{q}{2}\right)+S\left(-\frac{q}{2}\right)\right]^{2}\right\}\left|R \frac{\partial}{\partial R}\left(1+d \frac{\partial}{\partial R}\right) f_{m}(q)\right|^{2} \\
& +\frac{5}{48} K^{2} R^{2} R_{d}^{2} \Phi^{2}(q)\left[q R J_{|m|-1}(q R)-(|m|-1) J_{|m|}(q R)\right]^{2} \\
& -\frac{1}{2} K R R_{d} \Phi(q)\left\{1-\frac{1}{2}\left[S\left(\frac{q}{2}\right)+S\left(-\frac{q}{2}\right)\right]\right\}\left[q R J_{|m|-1}(q R)-(|m|-1) J_{|m|}(q R)\right] \\
& \quad \times \operatorname{Im}\left[\exp \left[2 i \sigma_{p}(R)\right] R \frac{\partial}{\partial R}\left(1+d \frac{\partial}{\partial R}\right) f_{m}^{*}(q)\right] .
\end{aligned}
$$

Expressions (39), (40) are allowed to contain the squared terms over the small values $R_{d} / R$ that guarantees the positivity of the cross-sections in the vicinities of minima.

Figure 1 shows the differential cross-section of the $110 \mathrm{MeV}$ deuterons elastically scattered by the ${ }^{208} \mathrm{~Pb}-$ nuclei. Figure 2 shows the differential cross-sections of the $80 \mathrm{MeV}$ deuterons scattered elastically and inelastically with the excitation of the first $2^{+}$state in the ${ }^{68} \mathrm{Zn}-$ nuclei. The differential cross-sections of the $80 \mathrm{MeV}$ deuteron dissociation with and without the excitation of the first $2^{+}$state in the ${ }^{68} \mathrm{Zn}-$ nuclei are presented on figure 3 .

\section{AMPLITUDE OF THE \\ THREE-NUCLEON-PARTICLE-NUCLEUS INTERACTION}

So far as the ${ }^{3} \mathrm{H}$-nucleus contains only one charged nucleon it is convenient to calculate the amplitude of the ${ }^{3} \mathrm{H}$-nucleus scattering with the excitation of low lying vibrational states of nuclei with the allowance for the Coulomb interaction, the inner structure of ${ }^{3} \mathrm{H}-$ nucleus, the finite values of nuclear surface diffuseness and surface refraction, with neglect of spins and isospins of the nucleons.

The amplitude of the ${ }^{3} \mathrm{H}$-nucleus-nucleus interaction is defined by formula [9]

$$
F(\mathbf{q})=\int d^{3} \rho d^{3} r \Psi_{f}^{*}(\boldsymbol{\rho}, \mathbf{r}) F(\mathbf{q}, \mathbf{w}, \mathbf{s}) \Psi_{0}(\boldsymbol{\rho}, \mathbf{r})
$$

where $\boldsymbol{\rho}=\mathbf{r}_{n 2}-\frac{1}{2}\left(\mathbf{r}_{n 1}+\mathbf{r}_{p}\right)$ and $\mathbf{r}=\mathbf{r}_{p}-\mathbf{r}_{n 1}$ are the coordinates of Jacoby; $\mathbf{r}_{n 1}, \mathbf{r}_{n 2}$ and $\mathbf{r}_{\mathbf{p}}$ are the nucleon radius-vectors; $\mathbf{w}$ and $\mathbf{s}$ are the projections of the vectors $\rho$ and $\mathbf{r}$ on the plane perpendicular to the incident ${ }^{3} \mathrm{H}$-beam direction ( $\mathrm{z}$-axis); $\mathbf{q}$ is the momentum transfer of the ${ }^{3} H$-nucleus center-of-mass; $\Psi_{0}(\rho, \mathbf{r})$ is the ${ }^{3} \mathrm{H}$-nucleus ground-state wavefunction; $\Psi_{f}(\rho, \mathbf{r})$ is the wavefunction of the related motion of the nucleons, which ${ }^{3} \mathrm{H}$-nucleus was consisted of, in the final state.

The amplitude $F(\mathbf{q}, \mathbf{w}, \mathbf{s})$ is equal to

$$
\begin{aligned}
F(\mathbf{q}, \mathbf{w}, \mathbf{s}) & =\frac{i K}{2 \pi} \int d^{2} b \exp (i \mathbf{q} \mathbf{b}) \\
& \times[1-\exp [2 i \chi(\mathbf{b}, \mathbf{w}, \mathbf{s})]]
\end{aligned}
$$

where $K$ is the projectile wavevector; $\mathbf{b}=\left(\mathbf{b}_{n 1}+\mathbf{b}_{n 2}+\right.$ $\left.\mathbf{b}_{p}\right) / 3$ is the impact parameter of the ${ }^{3} \mathrm{H}$-nucleus centerof-mass; $\mathbf{b}_{n 1}, \mathbf{b}_{n 2}$ and $\mathbf{b}_{p}$ are the nucleon impact parameters; $\chi(\mathbf{b}, \mathbf{w}, \mathbf{s})$ is the scattering phase of the ${ }^{3} \mathrm{H}$-nucleus.

The ${ }^{3} \mathrm{H}$-nucleus scattering phase has the form

$$
\chi(\mathbf{b}, \mathbf{w}, \mathbf{s})=\chi_{n 1}\left(\mathbf{b}_{n 1}\right)+\chi_{n 2}\left(\mathbf{b}_{n 2}\right)+\chi_{p}\left(\mathbf{b}_{p}\right)+\sigma_{p}\left(\mathbf{b}_{p}\right)
$$

where $\mathbf{b}_{n 1, p}=\mathbf{b}-\mathbf{w} / 3 \pm \mathbf{s} / 2, \mathbf{b}_{n 2}=\mathbf{b}+2 \mathbf{w} / 3$, $\chi_{n 1, n 2, p}\left(\mathbf{b}_{n 1, n 2, p}\right)$ are the nuclear parts of the nucleon 
scattering phase; $\sigma_{p}\left(\mathbf{b}_{p}\right)$ is the Coulomb part of the ${ }^{3} \mathrm{H}-$ nucleus' proton scattering phase.

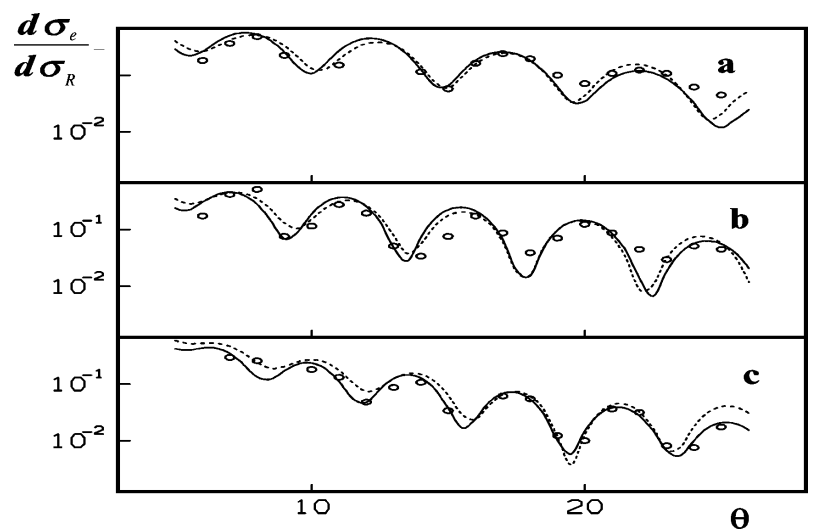

Fig. 5. The same as on Fig. 4 but for $217 \mathrm{MeV}$. Experimental data are taken from [2].

The amplitude $F(\mathbf{q}, \mathbf{w}, \mathbf{s})$ can be presented as a sum of the Coulomb scattering amplitude $F_{C}(\mathbf{q}, \mathbf{w}, \mathbf{s})$ and the nuclear scattering amplitude distorted by the Coulomb interaction $F_{N}(\mathbf{q}, \mathbf{w}, \mathbf{s})$ :

$$
F(\mathbf{q}, \mathbf{w}, \mathbf{s})=F_{C}(\mathbf{q}, \mathbf{w}, \mathbf{s})+F_{N}(\mathbf{q}, \mathbf{w}, \mathbf{s}),
$$

$$
F_{C}(\mathbf{q}, \mathbf{w}, \mathbf{s})=3 f_{C}\left(\frac{K}{3}, q\right) \exp (i \mathbf{q w} / 3+i \mathbf{q s} / 2)
$$

$$
\begin{gathered}
F_{N}(\mathbf{q}, \mathbf{w}, \mathbf{s})=\frac{i K}{2 \pi} \int d^{2} b \exp \left(i \mathbf{q} \mathbf{b}+2 i \sigma_{p}\left(\mathbf{b}_{p}\right)\right) \\
\times\left[1-\exp \left(2 i\left(\chi_{n 1}\left(\mathbf{b}_{n 1}\right)+\chi_{n 2}\left(\mathbf{b}_{n 2}\right)+\chi_{p}\left(\mathbf{b}_{p}\right)\right)\right)\right] \\
f_{C}\left(\frac{K}{3}, q\right)=-\frac{2}{3} K R^{2} \frac{n \Gamma(1+i n)}{\Gamma(1-i n)} \\
\times \frac{\exp (-2 i n \ln (3 q / 2 K))}{(q R)^{2}}
\end{gathered}
$$

$$
\exp \left(2 i \sigma_{p}\left(\mathbf{b}_{p}\right)\right)=\frac{\Gamma(1+K b / 3+i n)}{\Gamma(1+K b / 3-i n)}
$$

where $n=\frac{3 M Z e^{2}}{\hbar^{2} K}$ is the Coulomb parameter of the ${ }^{3} \mathrm{H}-$ nucleus proton; $M$ is the nucleon mass; $Z e^{2}$ is the nucleus charge.

The nucleon profile functions $\omega_{n 1, n 2, p}\left(\mathbf{b}_{n 1, n 2, p}\right)$ are introduced by the correlations

$$
\omega_{n 1, n 2, p}\left(\mathbf{b}_{n 1, n 2, p}\right)=1-\exp \left(2 i \chi_{n 1, n 2, p}\left(\mathbf{b}_{n 1, n 2, p}\right)\right)
$$

Therefore, the amplitude $F_{N}(\mathbf{q}, \mathbf{w}, \mathbf{s})$ acquires the form

$$
F_{N}(\mathbf{q}, \mathbf{w}, \mathbf{s})=\frac{i K}{2 \pi} \int d^{2} b \exp \left(i \mathbf{q} \mathbf{b}+2 i \sigma_{p}\left(\mathbf{b}_{p}\right)\right) \omega_{t}(\mathbf{b}, \mathbf{w}, \mathbf{s}),
$$

where the profile function $\omega_{t}(\mathbf{b}, \mathbf{w}, \mathbf{s})$ that describes the interaction between the ${ }^{3} \mathrm{H}-$ nucleus and the nucleus is equal to

$$
\begin{aligned}
& \omega_{t}(\mathbf{b}, \mathbf{w}, \mathbf{s})=\omega_{n 1}\left(\mathbf{b}_{n 1}\right)+\omega_{n 2}\left(\mathbf{b}_{n 2}\right)+\omega_{p}\left(\mathbf{b}_{p}\right)-\omega_{n 1}\left(\mathbf{b}_{n 1}\right) \omega_{p}\left(\mathbf{b}_{p}\right) \\
& -\omega_{n 2}\left(\mathbf{b}_{n 2}\right) \omega_{p}\left(\mathbf{b}_{p}\right)-\omega_{n 1}\left(\mathbf{b}_{n 1}\right) \omega_{n 2}\left(\mathbf{b}_{n 2}\right)+\omega_{n 1}\left(\mathbf{b}_{n 1}\right) \omega_{n 2}\left(\mathbf{b}_{n 2}\right) \omega_{p}\left(\mathbf{b}_{p}\right)
\end{aligned}
$$

The magnitudes $\omega_{n 1, n 2, p}\left(\mathbf{b}_{n 1, n 2, p}\right)$ are equal to (12). Using formulae (13)-(15), we find

$$
\omega_{t}(\mathbf{b}, \mathbf{w}, \mathbf{s})=\left\{1+\sum_{l, m} \alpha_{l, m} Y_{l, m}\left(\frac{\pi}{2}, \psi\right) R \frac{\partial}{\partial R}\right\} \omega^{(t)}(\mathbf{b}, \mathbf{w}, \mathbf{s})
$$

where $\omega^{(t)}(\mathbf{b}, \mathbf{w}, \mathbf{s})$ is defined by formula $(51)$.

The ${ }^{3} \mathrm{H}$-nucleus profile function $\omega^{(t)}(\mathbf{b}, \mathbf{w}, \mathbf{s})$ can be represented in the form

$$
\omega^{(t)}(\mathbf{b}, \mathbf{w}, \mathbf{s})=\left(1+\frac{3}{2} d \frac{\partial}{\partial R}+\frac{1}{2} d^{2} \frac{\partial^{2}}{\partial R^{2}}\right) \omega_{1}(\mathbf{b}, \mathbf{w}, \mathbf{s})+\omega_{2}(\mathbf{b}, \mathbf{w}, \mathbf{s})
$$


$\omega_{1}(\mathbf{b}, \mathbf{w}, \mathbf{s})=\frac{1}{3}\left[\omega_{n 1}\left(\mathbf{b}_{n 1}\right)+\omega_{n 2}\left(\mathbf{b}_{n 2}\right)+\omega_{p}\left(\mathbf{b}_{p}\right)\right]$,

$\omega_{2}(\mathbf{b}, \mathbf{w}, \mathbf{s})=\frac{1}{2}\left\{1-\frac{1}{3}\left[\omega_{n 1}\left(\mathbf{b}_{n 1}\right)+\omega_{n 2}\left(\mathbf{b}_{n 2}\right)+\omega_{p}\left(\mathbf{b}_{p}\right)\right]\right\}$

$\times\left\{\left[\omega_{n 1}\left(\mathbf{b}_{n 1}\right)-\omega_{p}\left(\mathbf{b}_{p}\right)\right]^{2}+\left[\omega_{n 2}\left(\mathbf{b}_{n 2}\right)-\omega_{p}\left(\mathbf{b}_{p}\right)\right]^{2}\right.$

$\left.+\left[\omega_{n 1}\left(\mathbf{b}_{n 1}\right)-\omega_{n 2}\left(\mathbf{b}_{n 2}\right)\right]^{2}\right\}$.

After the calculations similar to the ones presented in section II amplitude (41) acquires the form

$$
\begin{aligned}
F(\mathbf{q})= & F_{e}(\mathbf{q})+\sum_{l, m} \alpha_{l, m} Y_{l, m}\left(\frac{\pi}{2}, 0\right) F_{m}(\mathbf{q}) \\
F_{e}(\mathbf{q}) & =3 f_{C}(q) S_{1}(\mathbf{q}) \\
& +\frac{1}{3}\left\{S_{1}(\mathbf{q})+S_{2}(\mathbf{q})+S_{3}(\mathbf{q})\right\} f_{0}^{(N)}(\mathbf{q}) \\
& +i K R p \Phi(q) J_{0}(q R) \exp \left(2 i \sigma_{p}(R)\right) \\
F_{m}(\mathbf{q})= & \frac{1}{3}\left\{S_{1}(\mathbf{q})+S_{2}(\mathbf{q})+S_{3}(\mathbf{q})\right\} R \frac{\partial}{\partial R} f_{m}^{(N)}(\mathbf{q}) \\
& +i K R \Phi(q) p\left(q R J_{|m|-1}(q R)\right. \\
& \left.-(|m|-1) J_{|m|}(q R)\right) \exp \left(2 i \sigma_{p}(R)\right)
\end{aligned}
$$

where $f_{j}^{(N)}(\mathbf{q})=\left(1+\frac{3}{2} d \frac{\partial}{\partial R}+\frac{1}{2} d^{2} \frac{\partial^{2}}{\partial R^{2}}\right) f_{j}(\mathbf{q})$ and the notations introduced are

$$
\begin{gathered}
S_{1,2}(\mathbf{q})=\int d^{3} \rho d^{3} r e^{i \frac{\mathbf{q w}}{3} \pm i \frac{\mathbf{q s}}{2}} \Psi_{f}^{*}(\boldsymbol{\rho}, \mathbf{r}) \Psi_{0}(\boldsymbol{\rho}, \mathbf{r}), \\
S_{3}(\mathbf{q})=\int d^{3} \rho d^{3} r e^{-i \frac{2 \mathrm{q} w}{3}} \Psi_{f}^{*}(\boldsymbol{\rho}, \boldsymbol{r}) \Psi_{0}(\rho, \mathbf{r}), \\
p=\int d^{3} \rho d^{3} r p(\boldsymbol{\rho}, \mathbf{r}) \Psi_{f}^{*}(\boldsymbol{\rho}, \boldsymbol{r}) \Psi_{0}(\boldsymbol{\rho}, \mathbf{r}) ; \\
p(\boldsymbol{\rho}, \mathbf{r})=Q_{1}(\boldsymbol{\rho}, \mathbf{r})+Q_{2}(\boldsymbol{\rho}, \mathbf{r}), \\
Q_{1}(\boldsymbol{\rho}, \mathbf{r})=\rho \cos \theta_{1}+\frac{1}{2} r\left|\cos \theta_{2}\right|
\end{gathered}
$$

$$
\frac{1}{2} r\left|\cos \theta_{2}\right|<\rho \cos \theta_{1}
$$$$
Q_{2}(\boldsymbol{\rho}, \mathbf{r})=r\left|\cos \theta_{2}\right| ; \frac{1}{2} r\left|\cos \theta_{2}\right|>\rho \cos \theta_{1}
$$

The values of $\rho, \theta_{1}$ and $\theta_{2}$ are contained in the intervals: $0 \leq \rho \leq \infty, 0 \leq \theta_{1} \leq \pi / 2$ and $0 \leq \theta_{2} \leq \pi$.

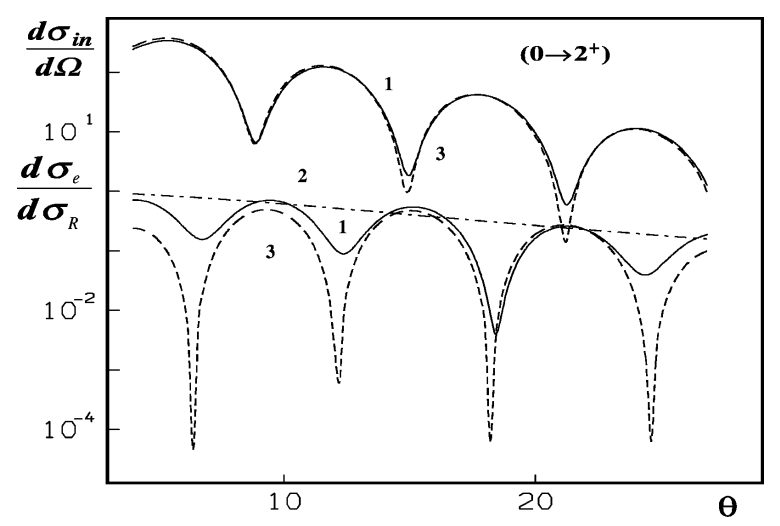

Fig. 6. The inelastic scattering differential cross-section $(\mathrm{mb} / \mathrm{sr})$ with the excitation of the first $2^{+}$state (the upper figure) and the ratio of the elastic scattering differential cross-section to the Rutherford one (the lower figure) for the $165 \mathrm{MeV}{ }^{3} \mathrm{H}-$ nuclei on the ${ }^{68} \mathrm{Zn}-$ nuclei $(R=5.7 \mathrm{fm}$, $d=0.60 \mathrm{fm}, \gamma=0.18, \beta_{2}=0.25$ ). Curves 1 show the interference between the Coulomb scattering and the nuclear one. Curve 2 is for pure Coulomb scattering. Curves 3 are for pure nuclear scattering.

\section{DIFFERENTIAL CROSS-SECTIONS OF THREE-NUCLEON-PARTICLE-NUCLEUS INTERACTION}

A complete set of the orthonormalized three-nucleonnucleus wavefunctions will be chosen in the form [8], [17]:

$$
\Psi_{0}(\boldsymbol{\rho}, \mathbf{r})=\psi_{0}(\boldsymbol{\rho}) \varphi_{0}(\mathbf{r})
$$

$$
\Psi_{\mathbf{u}}(\boldsymbol{\rho}, \mathbf{r})=\psi_{\mathbf{u}}(\boldsymbol{\rho}) \varphi_{0}(\mathbf{r})
$$

$$
\Psi_{\mathbf{u}, \mathbf{f}}(\boldsymbol{\rho}, \mathbf{r})=\psi_{\mathbf{u}}(\boldsymbol{\rho}) \varphi_{\mathbf{f}}(\mathbf{r})
$$

$$
\psi_{0}(\boldsymbol{\rho})=\sqrt{\frac{\lambda}{2 \pi}} \frac{\exp (-\lambda \rho)}{\rho}
$$




$$
\psi_{\mathbf{u}}(\boldsymbol{\rho})=\exp (i \mathbf{u} \boldsymbol{\rho})+\frac{1}{i u-\lambda} \frac{\exp (-i u \rho)}{\rho}
$$

where $\mathbf{u}=\left(2 \mathbf{k}_{\mathbf{n} 2}-\mathbf{k}_{\mathbf{d}}\right) / 3$ and $\mathbf{f}=\left(\mathbf{k}_{\mathbf{p}}-\mathbf{k}_{n 1}\right) / 2$ are the deuteron-neutron and deuteron's neutron-proton related motion wavevectors; $\mathbf{k}_{d}=\mathbf{k}_{n 1}+\mathbf{k}_{p}$ is the released deuteron wavevector; $\mathbf{k}_{n 1}, \mathbf{k}_{n 2}$ and $\mathbf{k}_{p}$ are the nucleon wavevectors in the final state; $\varphi_{0}(\mathbf{r})$ and $\varphi_{\mathbf{f}}(\mathbf{r})$ are defined by $(32),(33) ; \lambda=0.420 \mathrm{fm}^{-1}$. The wavefunctions (57)(59) correspond to the zero-value nuclear force range assumption (the $D$ - wave admixture to the ground-state is neglected) and, besides the ground state, describe the dissociation of three-nucleon-nucleus into two and three parts.

Taking into account that the variables are separated in the wavefunctions (57)-(59), the amplitudes (55) can be rewritten as

$$
\begin{aligned}
F_{e}(q) & =3 f_{C}\left(\frac{K}{3}, q\right) G_{1}(q / 3) G_{2}(q / 2) \\
& +\frac{1}{3}\left\{2 G_{1}(q / 3) G_{2}(q / 2)+G_{1}(2 q / 3)\right\} f_{0}^{(N)}(\mathbf{q}) \\
& +i K R \Phi(q) p J_{0}(q R) \exp \left(2 i \sigma_{p}(R)\right) \\
F_{m}(q) & =\frac{1}{3}\left\{2 G_{1}(q / 3) G_{2}(q / 2)\right. \\
& \left.+G_{1}(2 q / 3)\right\} R \frac{\partial}{\partial R} f_{m}^{(N)}(\mathbf{q}) \\
& +i K R \Phi(q) p\left(q R J_{|m|-1}(q R)\right. \\
& \left.-(|m|-1) J_{|m|}(q R)\right) \exp \left(2 i \sigma_{p}(R)\right)
\end{aligned}
$$

$$
\begin{gathered}
G_{1}(q)=\int d^{3} \rho e^{i \mathbf{q} \mathbf{w}} \psi_{f}^{*}(\boldsymbol{\rho}) \psi_{0}(\boldsymbol{\rho}) \\
G_{2}(q)=\int d^{3} r e^{i \mathbf{q s}} \varphi_{f}^{*}(\mathbf{r}) \varphi_{0}(\mathbf{r})
\end{gathered}
$$

The elastically and inelastically scattered amplitudes are defined by the magnitudes $G_{1}(q), G_{2}(q)$ and $p$ where the wavefunction (57) should be taken as $\Psi_{f}(\boldsymbol{\rho}, \mathbf{r})$ :

$$
\begin{gathered}
G_{1}(q)=\frac{1}{q R_{1}} \arctan \left(q R_{1}\right), \\
G_{2}(q)=\frac{1}{q R_{2}} \arctan \left(q R_{2}\right), \\
R_{1}=\frac{1}{2 \lambda}, \quad R_{2}=\frac{1}{2 \alpha}, \\
p=\frac{R_{1}}{12}\left[1+\frac{4 R_{1}}{R_{2}} \ln \left(1+\frac{R_{2}}{2 R_{1}}\right)\right] \\
+\frac{R_{2}}{6}\left[1+\frac{R_{2}}{4 R_{1}} \ln \left(1+\frac{2 R_{1}}{R_{2}}\right)\right]
\end{gathered}
$$

The differential cross-sections of the ${ }^{3} \mathrm{H}$-nucleus elastic scattering and inelastic scattering with the excitation of one-phonon vibrational states with spin $I$ in even-even nuclei are given by formulae (36). The differential cross-sections of the ${ }^{3} \mathrm{H}$-nucleus dissociation into the deuteron and neutron are defined by formulae (37) where we substitute $\mathbf{f} \rightarrow \mathbf{u}$. Finally, the differential cross-sections of the ${ }^{3} \mathrm{H}-$ nucleus dissociation into the proton and two neutrons have the form

$$
\begin{gathered}
\frac{d \sigma^{(t, n p n)}}{d \Omega}=\left|F_{0}(\mathbf{q}, \mathbf{u}, \mathbf{f})\right|^{2} \frac{d^{3} u}{(2 \pi)^{3}} \frac{d^{3} f}{(2 \pi)^{3}} \\
\frac{d \sigma^{(t, n p n)}(0 \rightarrow I)}{d \Omega}=\frac{\left|\beta_{I}\right|^{2}}{2 I+1} \sum_{m=-I}^{I}\left|Y_{I, m}\left(\frac{\pi}{2}, 0\right) F_{m}(\mathbf{q}, \mathbf{u}, \mathbf{f})\right|^{2} \frac{d^{3} u}{(2 \pi)^{3}} \frac{d^{3} f}{(2 \pi)^{3}}
\end{gathered}
$$

The angular distributions of the deuteron-neutron and neutron-proton-neutron systems center-of-masses, released in the ${ }^{3} \mathrm{H}$-nuclei dissociation are obtained with the help of completeness conditions for the functions (57)-(59):

$$
\begin{aligned}
& \frac{d \sigma^{(t, d n)}}{d \Omega}=D_{1}(q)\left|f_{C}(q)\right|^{2}+D_{2}(q)\left|f_{0}^{(N)}(q)\right|^{2}+D_{3}(q) \operatorname{Re} f_{0}^{(N)}(q)^{*} f_{C}(q) \\
& -K R \Phi(q) p J_{0}(q R)\left[D_{4}(q) I m f_{C}^{*}(q) \exp \left(2 i \sigma_{p}(R)\right)+D_{5}(q) I m f_{0}^{(N)}(q)^{*} \exp \left(2 i \sigma_{p}(R)\right)\right] K^{2} R^{2} \Phi^{2}(q) p_{1} J_{0}^{2}(q R)
\end{aligned}
$$




$$
\begin{aligned}
& \frac{d \sigma^{(t, d n)}(0 \rightarrow I)}{d \Omega}=\frac{\left|\beta_{I}\right|^{2}}{2 I+1} \sum_{m=-I}^{I}\left|Y_{I, m}\left(\frac{\pi}{2}, 0\right)\right|^{2} \sigma_{m}^{(t, d n)}(q) \\
& \sigma_{m}^{(t, d n)}(q)=D_{2}(q)\left|R \frac{\partial}{\partial R} f_{m}^{(N)}(q)\right|^{2}-K R \Phi(q) p D_{5}(q)\left(q R J_{|m|-1}(q R)-(|m|-1) J_{|m|}(q R)\right) \\
& \times \operatorname{Im} \exp \left(2 i \sigma_{p}(R)\right) R \frac{\partial}{\partial R} f_{m}^{(N)}(q)^{*}+K^{2} R^{2} \Phi^{2}(q) p_{1}\left(q R J_{|m|-1}(q R)-(|m|-1) J_{|m|}(q R)\right)^{2} \\
& \frac{d \sigma^{(t, n p n)}}{d \Omega}=T_{1}(q)\left|f_{C}(q)\right|^{2}+T_{2}(q)\left\{\left|f_{0}^{(N)}(q)\right|^{2}+9 \operatorname{Re} f_{0}^{(N)}(q)^{*} f_{C}(q)\right\} \\
& -K R \Phi(q) p J_{0}(q R) T_{3}(q) \operatorname{Im}\left\{\frac{9}{2} f_{C}^{*}(q)+f_{0}^{(N)}(q)^{*}\right\} \\
& \times \exp \left(2 i \sigma_{p}(R)\right)+K^{2} R^{2} \Phi^{2}(q) p_{2} J_{0}^{2}(q R) \\
& \frac{d \sigma^{(t, n p n)}(0 \rightarrow I)}{d \Omega}=\frac{\left|\beta_{I}\right|^{2}}{2 I+1} \sum_{m=-I}^{I}\left|Y_{I, m}\left(\frac{\pi}{2}, 0\right)\right|^{2} \sigma_{m}^{(t, n p n)}(q) \\
& \sigma_{m}^{(t, n p n)}(q)=T_{2}(q)\left|R \frac{\partial}{\partial R} f_{m}^{(N)}(q)\right|^{2}-K R \Phi(q) p T_{3}(q)\left(q R J_{|m|-1}(q R)-(|m|-1) J_{|m|}(q R)\right) \\
& \times \operatorname{Im} \exp \left(2 i \sigma_{p}(R)\right) R \frac{\partial}{\partial R} f_{m}^{(N)}(q)^{*}+K^{2} R^{2} \Phi^{2}(q) p_{2}\left(q R J_{|m|-1}(q R)-(|m|-1) J_{|m|}(q R)\right)^{2}, \\
& D_{1}(q)=9 G_{2}^{2}(q / 2)\left[1-G_{1}^{2}(q / 3)\right] \\
& D_{2}(q)=\frac{1}{9}\left\{1+4 G_{2}^{2}(q / 2)+4 G_{2}(q / 2) G_{1}(q)-\left[2 G_{2}(q / 2) G_{1}(q / 3)+G_{1}(2 q / 3)\right]^{2}\right\} \\
& D_{3}(q)=2 G_{2}(q / 2)\left\{2 G_{2}(q / 2)+G_{1}(q)-G_{1}(q / 3)\left[2 G_{2}(q / 2) G_{1}(q / 3)+G_{1}(2 q / 3)\right]\right\}, \\
& D_{4}(q)=6 G_{2}(q / 2)\left[1-G_{1}(q / 3)\right] \\
& D_{5}(q)=\frac{2}{3}\left\{1+2 G_{2}(q / 2)-2 G_{2}(q / 2) G_{1}(q / 3)-G_{1}(2 q / 3)\right\} \\
& p_{1}=-\frac{R_{2}^{2}}{96}-\frac{5 R_{1}^{2}}{12}+\frac{13 R_{1} R_{2}}{48}+\frac{R_{1}^{2}}{2}\left[\frac{3 R_{1}}{R_{2}}+\frac{1}{3}\right] \ln \left(1+\frac{R_{2}}{2 R_{1}}\right)+\frac{5 R_{2}^{3}}{96 R_{1}} \ln \left(1+\frac{2 R_{1}}{R_{2}}\right)
\end{aligned}
$$




$$
\begin{gathered}
+\frac{R_{2}^{3}}{16 R_{1}} \int_{1}^{\infty} \frac{d \xi}{\xi^{3}} \int_{1}^{\infty} \frac{d \zeta}{\zeta^{3}(\xi+\zeta)} \ln \left[1+\frac{2 R_{1}}{R_{2}}(\xi+\zeta)\right]-p^{2} \\
T_{1}(q)=9\left[1-G_{2}^{2}(q / 2)\right]\left[1-G_{1}^{2}(q / 3)\right], \\
T_{2}(q)=\frac{2}{9}\left[1-G_{1}^{2}(q / 3)\right]\left[1+G_{2}(q)-2 G_{2}^{2}(q / 2)\right], \\
p_{2}=\frac{3 R_{2}^{2}}{32}+\frac{3 R_{1}^{2}}{4}-\frac{13 R_{1} R_{2}}{48}-\frac{R_{1}^{2}}{2}\left[\frac{3 R_{1}}{R_{2}}+\frac{1}{3}\right] \ln \left(1+\frac{R_{2}}{2 R_{1}}\right)+\frac{7 R_{2}^{3}}{96 R_{1}} \ln \left(1+\frac{2 R_{1}}{R_{2}}\right) \\
-\frac{R_{2}^{3}}{16 R_{1}} \int_{1}^{\infty} \frac{d \xi}{\xi^{3}} \int_{1}^{\infty} \frac{d \zeta}{\zeta^{3}(\xi+\zeta)} \ln \left[1+\frac{2 R_{1}}{R_{2}}(\xi+\zeta)\right] \\
-\frac{R_{1}^{3}}{2 R_{2}} \int_{1}^{\infty} \frac{d \xi}{\xi^{3}} \int_{1}^{\infty} \frac{d \zeta}{\zeta^{3}(\xi+\zeta)} \ln \left[1+\frac{R_{2}}{2 R_{1}}(\xi+\zeta)\right]+p^{2} .
\end{gathered}
$$

\begin{tabular}{|c|c|c|c|c|c|c|c|c|c|}
\hline \multirow{2}{*}{ Nucleus } & & \multicolumn{6}{|c|}{ Composed ${ }^{3}$ He-nucleus } & \multicolumn{3}{|c|}{ Quasipoint ${ }^{3} \mathrm{He}-$ nucleus } \\
\cline { 3 - 10 } & $\mathrm{R}, \mathrm{fm}$ & $r_{0}, \mathrm{fm}$ & $\mathrm{d}, \mathrm{fm}$ & $\gamma$ & $\mathrm{R}, \mathrm{fm}$ & $r_{0}, \mathrm{fm}$ & $\mathrm{d}, \mathrm{fm}$ & $\gamma$ \\
\hline${ }^{90} \mathrm{Zr}$ & 130 & 6.3 & 1.41 & .60 & .20 & 6.45 & 1.44 & .55 & .45 \\
& 217 & 6.2 & 1.38 & .65 & -.3 & 6.30 & 1.41 & .55 & .15 \\
\hline${ }^{120} \mathrm{Sn}$ & 130 & 7.1 & 1.44 & .70 & .20 & 7.10 & 1.44 & .70 & .50 \\
& 217 & 6.9 & 1.40 & .60 & .10 & 7.00 & 1.42 & .55 & .40 \\
\hline${ }^{208} \mathrm{~Pb}$ & 130 & 8.2 & 1.38 & .60 & .00 & 8.20 & 1.38 & .60 & .50 \\
& 217 & 8.0 & 1.35 & .65 & .20 & 8.00 & 1.35 & .60 & .60 \\
\hline
\end{tabular}

Table. The diffraction model parameters for the ${ }^{3} \mathrm{He}-$ nuclei-nuclei elastic scattering differential cross-section $\left(R=r_{0} A^{1 / 3}\right)$.

Placing the charge of the ${ }^{3} \mathrm{He}-$ nucleus in its center-ofmass, we have carried out the analyses of experimental data on the elastic scattering of the 130 and $217 \mathrm{MeV}$ ${ }^{3} \mathrm{He}-$ nuclei on ${ }^{90} \mathrm{Zr},{ }^{120} \mathrm{Sn}$ and ${ }^{208} \mathrm{~Pb}$ nuclei (figures 4,5 and table).

Figure 6 shows the differential cross-section of the $165 \mathrm{MeV}{ }^{3} \mathrm{H}-$ nuclei scattered elastically and inelastically with the excitation of the first $2^{+}$state in the ${ }^{68} \mathrm{Zn}^{-}$ nuclei. The differential cross-sections of the $165 \mathrm{MeV}$ ${ }^{3} \mathrm{H}$-nuclei dissociation-into-two and -three-parts with and without excitation of the first $2^{+}$state in the ${ }^{68} \mathrm{Zn}-$ nuclei are presented on figures 7,8 .

\section{ANALYSIS OF EXPERIMENTAL DATA}

In [1]- [3] the complex optical potential with six fitting parameters was used to analyze the differential crosssections of the elastic scattering of the ${ }^{3} \mathrm{He}-$ nuclei by the ${ }^{90} \mathrm{Zr},{ }^{120} \mathrm{Sn}$ and ${ }^{208} \mathrm{~Pb}$ nuclei at 130 and $217 \mathrm{MeV}$ and deuterons by the ${ }^{208} \mathrm{~Pb}$ nuclei at $110 \mathrm{MeV}$. The optical model has enabled to obtain good agreement between the calculated and measured differential cross-sections. The results of the analysis have shown large depth of the real part of the potential $V$ as compared to the imaginary one $W: V / W \approx 8.4$ for $110 \mathrm{MeV}$ deuterons, $V / W \approx 4 \div 5$ for $130 \mathrm{MeV}$ and $V / W \approx 2 \div 3$ for the $217 \mathrm{MeV}{ }^{3} \mathrm{He}-$ nuclei. In other words, the optical potential in the considered case has a strong refraction and relatively small absorption. In the energy region under study this points on the existence of substantial non-diffraction effects the role of which increases noticeably with the increase of the scattering angle.

The diffraction model is known to describe the experimental data well when the absorption is large and the refraction is small. Unfortunately, this kind of experimental data is not available in literature now. In spite of that, as is seen from figures 1, 2, 4, 5 and the Table, the diffraction method developed by us, being a small scattering angle approach and having only three fitting 
parameters, explains the given data well enough. We emphasize that in our approach the diffraction theory enables us to obtain the closed-form expressions for the differential cross-sections.

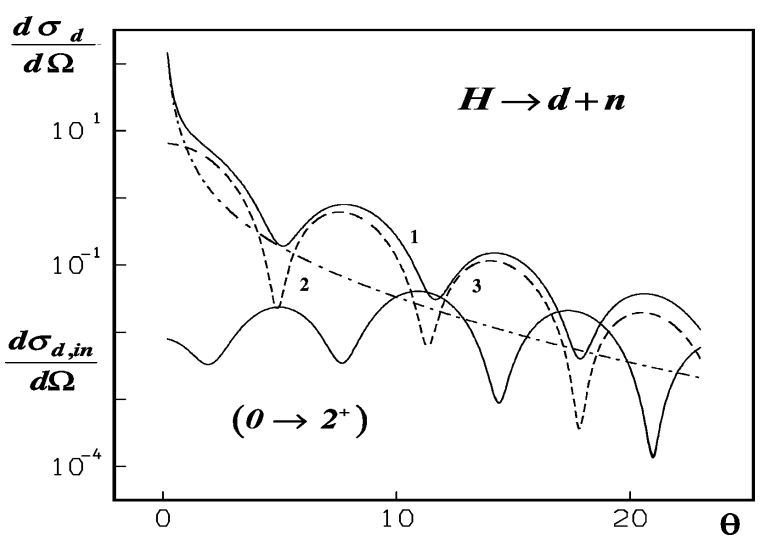

Fig. 7. The differential cross-section $(b / s r)$ of the $165 \mathrm{MeV}$ ${ }^{3} \mathrm{H}$-nuclei dissociated into two parts without (the upper figure) and with the excitation of the first $2^{+}$state (the lower figure) of the ${ }^{68} \mathrm{Zn}$-nuclei ( $R=5.7 \mathrm{fm}, d=0.60 \mathrm{fm}$, $\left.\gamma=0.18, \beta_{2}=0.25\right)$ as a function of the deuteron-neutron center-of-mass scattering angle $\theta$ (deg.). Curve 1 shows the interference between the Coulomb dissociation and the nuclear one. Curve 2 is for pure Coulomb dissociation. Curve 3 is for pure nuclear dissociation.

The quasipoint-projectile assumption for the ${ }^{3} \mathrm{He}-$ nucleus (assuming that the nucleons comprising the ${ }^{3} \mathrm{He}-$ nucleus are placed in its center of mass) leads to greater values of $R$ for ${ }^{90} \mathrm{Zr}$ as compared with the case where the ${ }^{3} \mathrm{He}$ inner structure is considered. The values of $R$ for the heavier nuclei remain almost the same. The values of $d$ slightly decrease. The refraction parameter $\gamma$ substantially increases for the quasipoint ${ }^{3} \mathrm{He}-$ nucleus. This fact is not surprising if we bear in mind that the optical model which does not consider the inner structure of the projectile points on large refraction too.

The differential cross-section of the inelastic deuteron and the ${ }^{3} \mathrm{H}$-nucleus scattering with excitation of low lying vibrational states of nuclei obeys the diffraction rule of phases [18], [19]: this cross section oscillates in phase with the cross-section of elastic scattering if $I$ is odd, and out of phase if $I$ is even (figures 2, 6). Figures 3, 7, 8 show that the same rule is valid for the cross-sections of the deuteron and ${ }^{3} \mathrm{H}$-nucleus dissociation-into-two and -three-parts with and without the excitation of low lying vibrational states of nuclei.

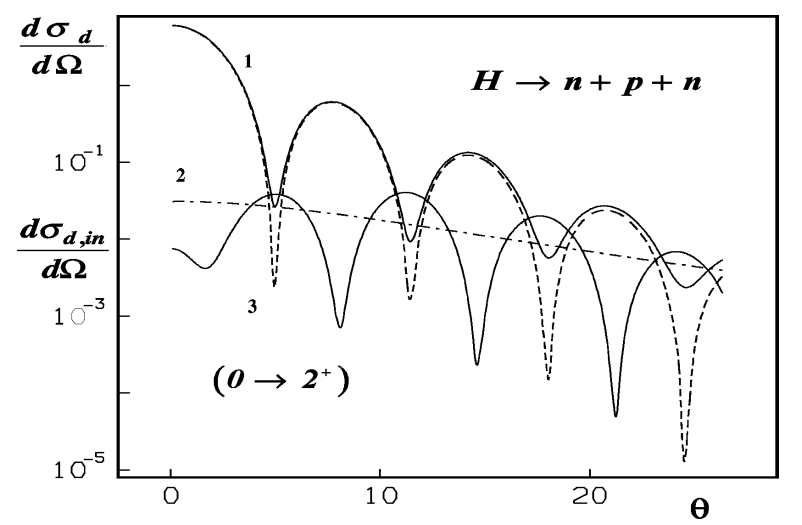

Fig. 8. The differential cross-section $(b / s r)$ of the $165 \mathrm{MeV}$ ${ }^{3} \mathrm{H}$-nuclei dissociated into three parts without (the upper figure) and with the excitation of the first $2^{+}$state (the lower figure) of the ${ }^{68} \mathrm{Zn}$-nuclei ( $R=5.7 \mathrm{fm}, d=0.60 \mathrm{fm}, \gamma=0.18$, $\left.\beta_{2}=0.25\right)$ as a function of the neutron-proton-neutron center-of-mass scattering angle $\theta$ (deg.). Curve 1 shows the interference between the Coulomb dissociation and the nuclear one. Curve 2 is for pure Coulomb dissociation. Curve 3 is for pure nuclear dissociation.

The differential cross-sections of the deuteron and the ${ }^{3} \mathrm{H}$-nucleus dissociation-into-two and -three-parts are the oscillating functions of the scattering angle (momentum transfer) of the center-of-masses (figures $3,7,8$ ). Figures 2, 3, 6-8 show that the cross-sections of elastic scattering and dissociation oscillate in phase proving the quasielastic character of the dissociation processes. The Coulomb interaction consideration exerts a substantial influence on the values of elastic scattering and dissociation cross-sections (figures 1-3,6-8). Taking into account the Coulomb interaction is especially important in calculating the deuteron and ${ }^{3} \mathrm{H}-$ nucleus dissociationinto-two and -three-parts where the Coulomb dissociation predominates in the region of small scattering angles of the released nucleon system center-of-mass.

Therefore, the many-nucleon-particle inner structure consideration allows to obtain the realistic values for fitting parameters on the basis of the diffraction analysis of experimental data. The detailed experiments on the elastic scattering of complex nuclei by different nuclei at different energies and further theoretical analysis will give more precise information about the mechanisms of interaction of the colliding particles and their inner structure.

\section{ACKNOWLEDGMENT}

The research described in this publication was supported, in part, by the State Fund of Fundamental Researches of Ukraine under Grant No. 2.4/416. 
[1] N. Willis, I. Brissand, Y. Le Bornec, B. Taticheff, G. Duhamel, Nucl. Phys. A 204, 454 (1973).

[2] A. Djaloeis, J.-P. Didelez, A. Golonsky, W. Oelert, Nucl. Phys. A 306, 221 (1978).

[3] A. C. Betker, C. A. Gagliardi, D. R. Semon, R. E. Tribble, H. M. Xu, A. F. Zaruba, Phys. Rev. C 48, 2085 (1993).

[4] R. J. Glauber, Phys. Rev. 99, 1515 (1955).

[5] R. J. Glauber, Phys. Rev. 100, 241 (1955).

[6] V. Franco, R. J. Glauber, Phys. Rev. 142, 1195 (1966).

[7] A. I. Akhiezer, A. G. Sitenko, Phys. Rev. 106, 1236 (1957).

[8] Yu. A. Berezhnoy, A. P. Soznik, Sov. J. Nucl. Phys. 9, $760(1969)$.

[9] Yu. A. Berezhnoy, A. P. Soznik, Sov. J. Nucl. Phys. 29, 350 (1979).

[10] Yu. A. Berezhnoy, V. Yu. Korda, Int. J. Mod. Phys. E 3, 149 (1994).
[11] G. Fäldt, Phys. Rev. D 2, 846 (1970).

[12] T. E. O. Ericson, in Preludes in Theoretical Physics, edited by A. de Shalit, H. Feshbach, L. van Hove (NorthHolland, Amsterdam, 1965) p. 321.

[13] W. E. Frahn, Diffractive Processes in Nuclear Physics (Clarendon Press, Oxford, 1985).

[14] W. E. Frahn, R. H. Venter, Ann. Phys. (Paris) 24, 243 (1963).

[15] W. E. Bassiches, A. Dar, Ann. Phys. (Paris) 36, 130 (1966).

[16] S. Varma, A. Dar, Ann. Phys. (Paris) 39, 435 (1966).

[17] J. M. Knight, J. S. O'Connel, F. Prats, Phys. Rev. 164, 1354 (1967)

[18] J. S. Blair, Phys. Rev. 115, 928 (1959).

[19] N. Austern, J. S. Blair, Ann. Phys. (Paris) 33, 15 (1965).

[20] G. Duhamel, L. Marcus, H. Langevin-Joliot, J. P. Didelez, P. Narboni, C. Stephan, Nucl. Phys. A 174, 485 (1971).

\section{ДИФРАКЦЙНА ВЗАЄМОДІЯ ДЕЙТРОНІВ І ТРИНУКЛОННИХ ЯДЕР З ВА ЖКИМИ Я ДРАМИ}

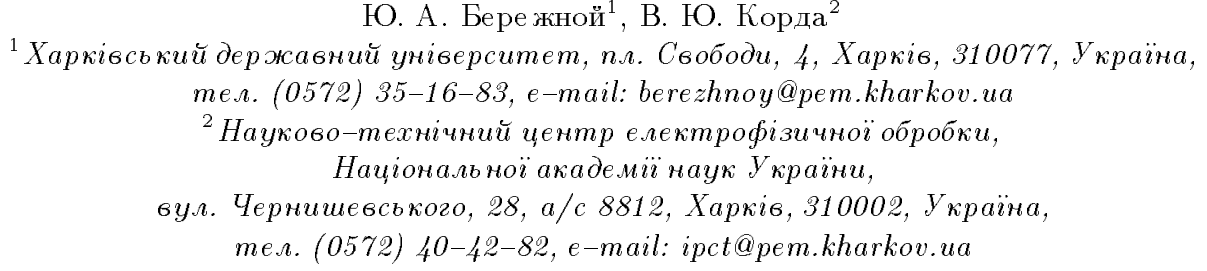

Розроблено новий дифракційний підхід, який дозволив дістати аналітичні вирази для диференціяльних перерізів пру жного й непру жного розсіяння та розщеплення на дві та три частини дво- і тринуклонних ядер ва жкими ядрами. Диференціяльні перерізи розщеплення виявляються осцилюючими функціями кута вильоту центра мас частинок, що налітають. Проведено аналіз експериментальних даних 3 пру жного $\mathbf{i}$ непру жного розсіяння дейтронів і ядер ${ }^{3} \mathrm{He}$ промі жних енергій ва жкими ядрами. Результати розрахунків добре узгоджуються з експериментальними даними для всіх використаних ядер-мішеней. 